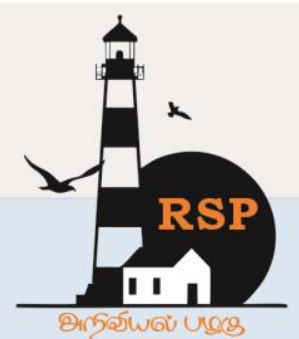

INTERNATIONAL RESEARCH JOURNAL ON ADVANCED SCIENCE HUB

Special Issue of First International Conference on Management, Science and Technology (ICMST 2021)

\title{
Quantum Computing: Applications and Future Importance
}

\author{
W. Sarada ${ }^{1}$, N. Rajalaxmi ${ }^{2}$, G. Suchitha Reddy ${ }^{3}$
}

${ }^{1,2,3}$ Assistant Professor, Department. of Computer Science, RBVRR Women's College, Narayanaguda, Hyderabad, Telangana.

saradaw20012011@gmail.com ${ }^{1}$

\begin{abstract}
Quantum computing is a fantastic technology that has been evolved throughout time and is 100 trillion times quicker than a supercomputer. It is based on quantum theory, which can do calculations using probability theory and linear algebra. These two disciplines of research are involved in all sub-areas of Data Science and other contemporary technology domains, and can answer a wide range of complex problems which even the most powerful supercomputer cannot carry out. Instead of just O's and 1's where the classical computers encipher the data or convert the information, it works on qubit that is, it can hold up to two bits which is called as Quantum superposition where it has two different states zero or one in which it can be defined and is also possible for it to exist in both, i.e. a superposition of the two states. Light based quantum computer outperforms the classical super computer by exceeding its performance speed. Quantum computing can solve known problems such as encryption and cyber security. Quantum computing has the ability to process quickly to arrive at a solution and offers well and more computing power to the world of Artificial Intelligence systems and efficient Machine learning systems by solving small and large problems much faster. The main aim or objective of this paper is to discuss about the applications and importance of quantum computing in future which is very helpful to the people who are associated with cryptography, Aviation, Data Analytics, Forecasting, Medical research, Pattern matching, self-driving cars and so on.
\end{abstract}

Keywords: Quantum computing, Supercomputer, Applications, Importance.

\section{Introduction}

Quantum computers have three states 0 or 1 or both 0 and 1 . It exists in two places at the same time and can even teleport. Both Quantum and classical super computers make an effort to solve problems, but the way they handle or control the data to get solutions is primarily distinct. In this part we are trying to give a description of the way the quantum computers appear different compared to the classical supercomputers by interpolating two fundamental principles of quantum mechanics which are crucial for their action of functioning, Superposition which is the contradictory ability of a quantum entity or an object, like an electron, to concurrently exist in several states and entanglement which means it is the process of substituting of quantum study of facts or data or knowledge obtained from investigation at a length of the space between two points [2]. Bits in classical computing are transistors that work on by controlling the flow of the electrical current that can be off or on, corresponding to the states 0 and 1. Assimilation or interpretation of superposition makes it possible to comprehend the basic part of findings in quantum computing, the qubit or the quantum bit with contrasting probabilities that can be controlled or influenced by quantum move during calculations.[1-4]. 


\section{Types of Operational Frameworks}

There are several types. One of the best recognized frameworks is the logical or gate-based quantum computing depending up on the qubit type, the initial states are a series of gate operations such as current or laser pulses subjected to AND, OR, and NOT gates of classical computation which are then measured and outcome obtained [4]. The second one is measurement-based computation in which entangled qubits serve as the initial state, based on the outcome, furthermore measures are carried out on other qubits and subsequently the solution is reached. The third one is an attractive approach which is topological quantum computation which are yet to be manifested, where qubits and their actions are based on quasiparticles and their queue operations as these systems are subjectively safeguarded against noise, which destroys the consistency of other qubits. Last one is the analog quantum computers or quantum simulators which can be thought of as specific purpose quantum computers conceived by Feynman [1] programmed as model quantum systems having the capability to intent a query like in very high temperatures in what manner the superconductors work, by what means certain chemicals react, in what way materials are designed based on certain properties.

\section{Type of Applications}

There are some areas of application which are suitable with respect to quantum computing to study, research, investigate and survey. It depends on how the Organizations or enterprising entities engaged in commercial, industrial, or professional activities can take expedience of this advancement in quantum computing. One of the area's most people who are associated with is the advanced cryptography where the classical computers are impractical to break the cipher text that uses very huge prime decomposition. With the quantum computers it is faster and feasible to protect the digital lives and assets. Another area is Aviation where the Quantum skills, methods, and processes enable much more complicated Computer simulations that are run according to such programs which can produce knowledge out of reach of mathematical analysis or natural experimentation like aeronautical scenarios or outline which helps in the routing and scheduling of aircrafts having immense trading benefits for time and costs.[5-8].

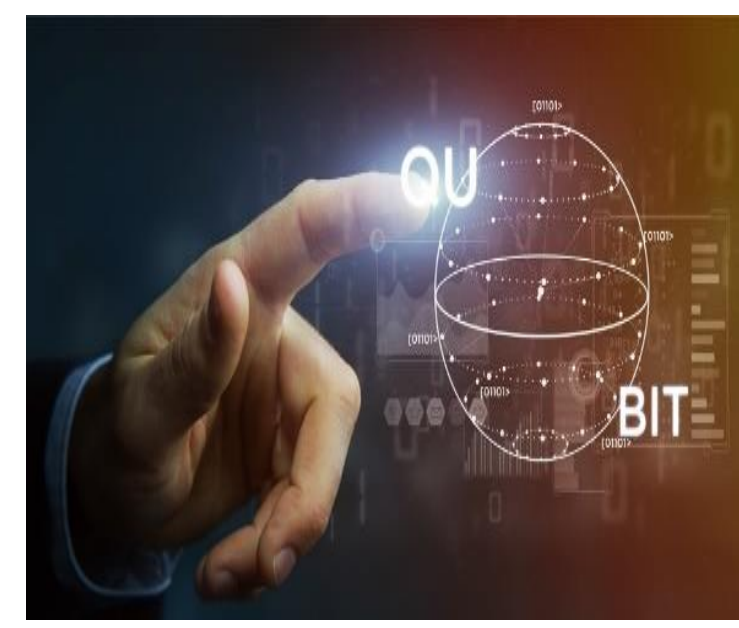

Fig.1: Applications of Quantum Computing

Giant companies like Airbus and Lockheed Martin diligently investigating and putting money, effort, time, etc. to take additional benefit of the power of computing and maximizing the capacity to develop into something in the future. Third one is the Data Analytics where using quantum computing can resolve problems on a large scale. An area of study of analysing topologically the behaviour of geometrical shapes in a particular way give the details of calculations that are simply not feasible with today's traditional computers with respect to the data set used. With quantum computing which promises relatively simple calculations. NASA is eyeing at using quantum computing for examining the vast amount of information they pile up about the space, as well as investigate effective and safer strategies of space travel. Fourth one is about forecasting weather etc., using quantum computing forecasting can be very accurate and can derive economically additional benefits when compared to classical computing were adding too many factors takes longer simulation time.Fifth one is Matching Patterns, with quantum computing prognosticating the way in which the complex traffic patterns are going to change so that for example Volkswagen is presently eyeing to notify the drivers regarding the traffic scenario 45 minutes in advance. Sixth one is Medical research, with quantum computing which enables the chemists to identify and detect something new quickly leading to cure for an extent of diseases by reducing costs and time [3]. Last one is about SelfDriving Cars where car companies like Tesla and Technology companies such as Apple and Google are industriously expanding driverless cars by improving the living standards of people, reducing pollution and congestion with several other 
www.rspsciencehub.com

additional benefits. Using quantum computing Volkswagen maximized traffic flow and presently Google and VW are using quantum computers for developing batteries, in transportation and selfdriving technology.[9-11].

\section{Importance of Quantum Computing}

In order to adequately process large volumes of data created every day and to extract meaningful information from it requires much more computing power this is where the quantum computers step in to solve problems in no time which are impossible for the classical or traditional computers which take billions of years to solve [4].
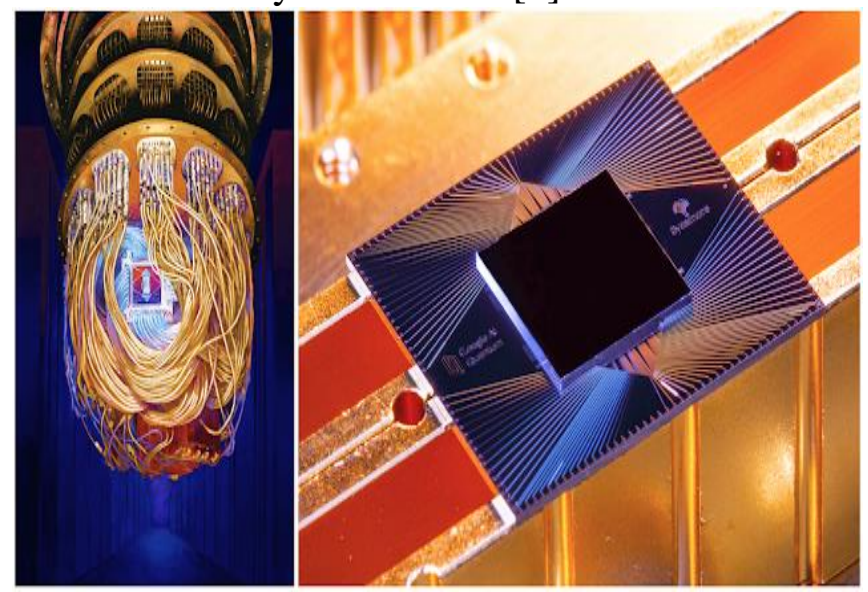

Fig.2: Photograph of the Googles Sycamore Processor

Googles quantum computer Sycamore contains 58 qubits which makes about 158 million times faster than the world's fastest supercomputer. The quantum computer accomplished the complex computation in 200 seconds. IBM's current quantum computer, contains 65 qubits. Its ambitious goal is to build one quantum computer containing 1000 qubits by 2023 and by 2025, frictionless quantum computing will begin to be a reality. Quantum computers will change the perspective of data security and would be able to crack today's several encryption techniques, predictions are that it would create replacements which are resistant to unauthorized access or infiltration by a computer hack. The intent is not to replace classical computers which are better at some tasks such as email, spreadsheets, DTP etc. Quantum computers are good at solving optimization problems determining the simplest delivery routes, schedule flights at an airport then on and process the quantity of knowledge generated within the age of massive data. 2.5 Exabyte's of data is being produced on a daily basis which is identical or almost like the content on 5 million laptops.

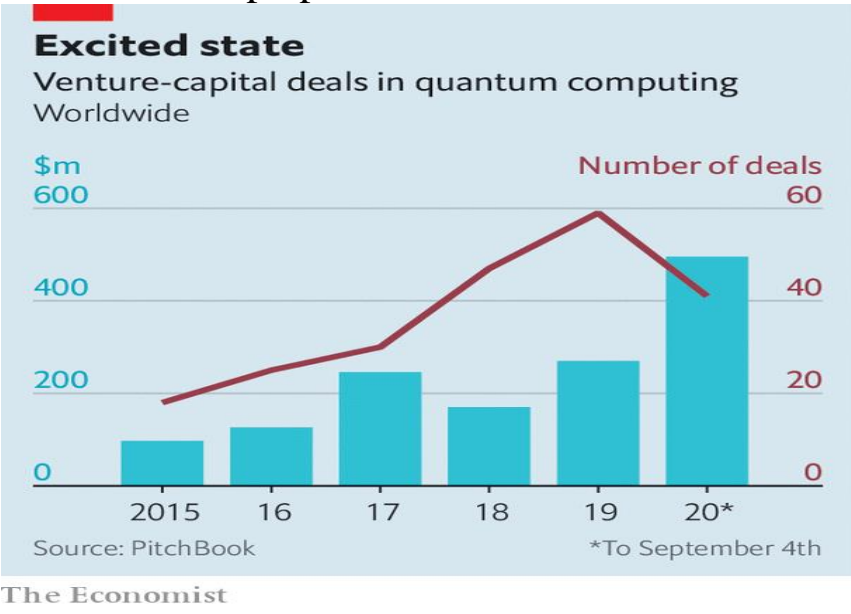

Fig.3: Commercializing Quantum Computers

As stated by some estimates or apprises, in 2020 it's worth \$5 million dollars the Quantum Computing market is anticipated to grow from USD 472 million in 2021 to USD 1,765 million by 2026, at a CAGR of $30.2 \%$. Currently, companies can buy the D-Wave quantum computer precedent in use by Lockheed Martin, Airbus, Volkswagen and other voyagers in this space. We might not see for a few times the total prospective of quantum computing but, the initial research and interoperability are showing incredible future benefits.

\section{Advantages and Downsides \\ 5.1 Advantages}

$>$ Power consumption reduced from 100 to 1000 times.

$>$ Able to calculate 1 trillion moves per second.

$>$ Works with quantum bits which might be in 'superpositions of states'.

\subsection{Disadvantages}

$>$ To keep them stable, they have to be cold.

$>$ They are very fragile

$>$ Difficult to create, engineer and program

\section{Conclusion}

In the majority, usually, any application which requires a lot of computations tending to strengthen or increase especially for a classical super computer is seemingly qualified as an affable prospect for a quantum computer. Quantum computing remains within the early stage within the development or growth which implies it'll become better or more successful as time passes over the subsequent few years. Within the fiscal year 2021 budget the Indian government has 


\section{www.rspsciencehub.com}

allocated INR 8,000 crore, to be spent over the following five years on the National Mission on Quantum Technologies and Application.

\section{References}

[1].Feynman RP. Simulating physics with computers. International Journal of Theoretical Physics.1982; 21(6-7):467-488.

[2].Aaronson, S. (2008). The limits of quantum computers. Scientific American.Monz, T., Nigg, D., Martinez, E. A., Brandl, M. F., Schindler, P., Rines, R., \& Blatt, R. (2016).

[3].Mannhold, R., Kubinyi, H., \& Folkers, G. (2006). Quantum medicinal chemistry (Vol. 17). John Wiley \& Sons.

[4].Aaronson, S.: Lower bounds for local search by quantum arguments. SIAM J. Comput. 35(4), 804-824 (2006).

[5].H Nishimura, T Yama kami - Polynomial time quantum computation with advice. Information Processing Letters, 2004 - Elsevier.
Volume 03 Issue 06S June 2021

[6].H Klauck, R Špalek, R De Wolf Quantum and classical strong direct product theorems and optimal Time-space trade-off - SIAM Journal on Computing, 2007 - SIAM.

[7].S Aaronson Quantum copy Protection and Quantum money- 2009, 24th Annual IEEE Conference on, 2009 - ieeexplore.ieee.org.

[8].A Ambainis, A Nayak, A Ta-Shma Dense quantum coding and Quantum finite automata Journal of the ACM (JACM, 2002 dl.acm.org.

[9].R Raz Quantum information and the PCP theorem - 46th Annual IEEE Symposium on Foundations of 2005 - ieeexplore.ieee.org.

[10].S Aaronson, A Drucker - A full Characterization of quantum Advice - SIAM Journal on Computing, 2014 - SIAM

[11].https://www.ncbi.nlm.nih.gov/Books/NBK53 8701/ 